\title{
Interim Tumor Progression and Volumetric Changes of Surgical Cavities during the Surgery-to-Radiotherapy Interval in Anaplastic Gliomas: Implications for Additional Pre-radiotherapy Magnetic Resonance Imaging
}

\author{
Chan Woo Wee, MD, PhD ${ }^{1,2}$ \\ II Han Kim, MD, PhD 2,3 \\ Chul-Kee Park, MD, PhD 3,4 \\ Jin Wook Kim, MD, PhD 3,4
}

${ }^{1}$ Department of Radiation Oncology, SMG-SNU Boramae Medical Center, Seoul, ${ }^{2}$ Department of Radiation Oncology, ${ }^{3}$ Cancer Research Institute, ${ }^{4}$ Department of Neurosurgery, Seoul National University College of Medicine, Seoul, Korea

\begin{abstract}
Purpose
This study was designed to investigate the incidence of interim disease progression (IPD) and volumetric changes of the surgical cavity (SC) during the surgery-to-radiotherapy interval (SRI), and eventually assess the value of magnetic resonance imaging (MRI) at the time of radiotherapy $(\mathrm{RT})$ planning in newly diagnosed anaplastic gliomas.
\end{abstract}

\section{Materials and Methods}

Among 195 anaplastic glioma patients who underwent RT, 121 were evaluable with two separate MRIs during SRI. The presence of IPD was determined using the updated Response Assessment in Neuro-Oncology size criteria. In 84 patients who underwent surgical resection, each $\mathrm{SC}$ was contoured by a radiation oncologist and the volumetric changes of the SCs were calculated between the two separate MRIs. Daily rate of change in the SC volume was calculated assuming an exponential and linear change.

\section{Results}

Five of 121 patients (4.13\%) demonstrated IPD during SRI, and the incidence was significantly higher in patients undergoing biopsy (vs. surgical resection, $12.9 \%$ vs. $1.1 \%, p=0.015$ ) and in patients with remnant contrast-enhancing tumor after surgery (15.8 vs. $2.0 \%$, $\mathrm{p}=0.027)$. The mean daily rate of absolute change in SC was 1.06\% (95\% confidence interval [Cl], 0.89 to 1.23$)$ and $0.89 \%(95 \% \mathrm{Cl}, 0.77$ to 1.02$)$ according to the exponential and linear model, respectively. The expected mean volumetric change at 2 weeks were $16.64 \%$ (95\% Cl, 13.77 to 19.52) and 12.51\% (95\% Cl, 10.77 to 14.26), respectively.

\section{Conclusion}

IPD during the SRI is rare in surgically resected anaplastic gliomas. However, pre-RT MRI is essential for accurate RT-target delineation and disease evaluation for patients initiating RT beyond postoperative 2 weeks and undergoing biopsy, respectively.

\author{
Cancer Research Institute, Seoul National \\ University College of Medicine, 101 Daehak-ro, \\ Jongno-gu, Seoul 03080, Korea \\ Tel: 82-2-2072-2528 \\ Fax: 82-2-765-3317 \\ E-mail: ihkim@snu.ac.kr \\ Received September 9, 2019 \\ Accepted October 31, 2019 \\ Published Online October 31, 2019
}

Correspondence: Il Han Kim, MD, PhD

\author{
Key words \\ Anaplastic glioma, Radiotherapy, Interim progression, \\ Surgical cavity, Magnetic resonance imaging
}

\section{Introduction}

Anaplastic gliomas (AG) are rare malignant brain tumors consisting of only $10 \%-15 \%$ of all newly diagnosed gliomas [1]. This nomenclature normally refers to anaplastic astrocytoma (AA), anaplastic oligodendroglioma (AO), or anaplastic oligoastrocytoma (AOA). These tumors are all categorized as grade III by the 2016 World Health Organization classification [2]. However, the standard treatment of these tumors following surgical resection or biopsy varies according to the histologic classification as well as the molecular variation $[3,4]$. Furthermore, the median survival after standard treatment vary ranging 3-15 years depending on molecular and histological subtypes [5-7].

Nevertheless, unlike grade II gliomas, radiotherapy (RT) is universally recommended in AGs regardless of type of surgery or combination with chemotherapy $[3,4]$. Target delineation is a critical step in RT planning which begins with the delineation of gross target volumes encompassing the surgi- 
cal cavity (SC) and remnant tumor. Since brain tissues are well-visualized on magnetic resonance imaging (MRI) compared to computed tomography (CT) imaging, clinicians [8] and prospective trials [5-7] have required MRIs to be fused to the simulation CT for target delineation and RT planning. In some studies, only preoperative MRI were mandatory, and although guidelines recommend postoperative MRIs to be used for RT-target delineation [3,4], the optimal timing of postoperative MRI used for RT planning is unknown. Concerns of interim disease progression (IPD) as well as changes in anatomical geometries during the surgery-to-radiotherapy interval (SRI) exist.

Recently, Wee et al. [9] have reported a significant incidence of $19.3 \%$ of IPD using two separate MRIs taken during the SRI in glioblastoma patients, and have recommended the routine use of additional pre-RT MRIs for disease evaluation and accurate RT-target delineation. The rapid growth of glioblastoma in a short-term interval has been confirmed by other investigators as well [10-14]. However, no study has investigated on the growth dynamics of AGs, particularly, the incidence of IPD during SRI. Therefore, it is unknown whether the routine use of additional pre-RT MRI in AGs can be justified.

In the current study, we investigated the incidence of IPD in AGs and the volumetric changes of SCs in surgically resected patients to evaluate the necessity of pre-RT MRIs for accurate RT-target delineation.

\section{Materials and Methods}

\section{Patients and treatment}

Patients who were histologically diagnosed as AA, AO, or AOA during 2007-2016 were included in this study. Patients who received pre-RT chemotherapy were excluded. Among 195 AG patients, 121 patients who underwent an additional MRI for RT planning at the time of simulation CT were further selected for the study. Baseline postoperative MRI was done in all patients within 48 hours from surgery except for those undergoing biopsy only. All MRIs consisted of the following sequences at least: T1 weighted images with and without gadolinium-contrast enhancement; T2 weighted images with or without fluid-attenuated inversion recovery sequences. The clinical characteristics of patients and treatments are summarized in Table 1. The median prescribed RT dose was $61.2 \mathrm{~Gy}$ in 34 fractions. Fifty-three patients (53/121, $43.8 \%$ ) received chemotherapy concurrently and/or post-RT.
Table 1. Patient and treatment characteristics

\begin{tabular}{|lc|}
\hline Variable & No. $(\%)$ \\
\hline Total & $121(100)$ \\
\hline Age, median (range, yr) & $43(18-71.5)$ \\
\hline Sex & \\
\hline Male & $65(53.7)$ \\
\hline Female & $56(46.3)$ \\
\hline ECOG PS & \\
\hline $0-2$ & $118(97.5)$ \\
\hline 3 & $3(2.5)$ \\
\hline Histology & $81(66.9)$ \\
\hline AA & $26(21.5)$ \\
\hline AO & $14(11.6)$ \\
\hline AOA & \\
\hline Contrast enhancement ${ }^{\text {a) }}$ & $56(46.3)$ \\
\hline Yes & $65(53.7)$ \\
\hline No & \\
\hline Surgeryb) & $64(52.9)$ \\
\hline GTR & $26(21.5)$ \\
\hline PR & $31(25.6)$ \\
\hline Biopsy & $68(56.2)$ \\
\hline Chemotherapy ${ }^{\text {c }}$ & $33(27.3)$ \\
\hline None & $5(4.1)$ \\
\hline Concurrent \pm adjuvant TMZ & $15(12.4)$ \\
\hline Adjuvant TMZ & \\
\hline Adjuvant PCV & \\
\hline
\end{tabular}

ECOG PS, Eastern Cooperative Oncology Group performance status; AA, anaplastic astrocytoma; AO, anaplastic oligodendroglioma; AOA, anaplastic oligoastrocytoma; GTR, gross total resection; PR, partial resection; TMZ, temozolomide; PCV, procarbazine/lomustine/vincristine. a) On preoperative diagnostic magnetic resonance imaging, ${ }^{b}$ On postoperative magnetic resonance imaging (within 48 hours), 'sequence of chemotherapy regarding radiotherapy.

\section{Interim disease progression}

The development of IPD during SRI was evaluated using the two separate MRIs including the MRI taken at the time of RT planning CT and the following: (1) in patients undergoing surgical resection: postoperative MRI within 48 hours of surgery; (2) in patients undergoing biopsy: navigation MRI at the time of surgery. The size criteria from the 'updated Response Assessment in Neuro-Oncology (RANO) criteria for high-grade gliomas' were used to evaluate disease progression as previously described $[9,15]$. For non-enhancing tumors, increase over $25 \%$ of the sum of the perpendicular diameters from the lesion with T2 high signal intensity was designated as IPD. Risk factors of IPD were evaluated. 


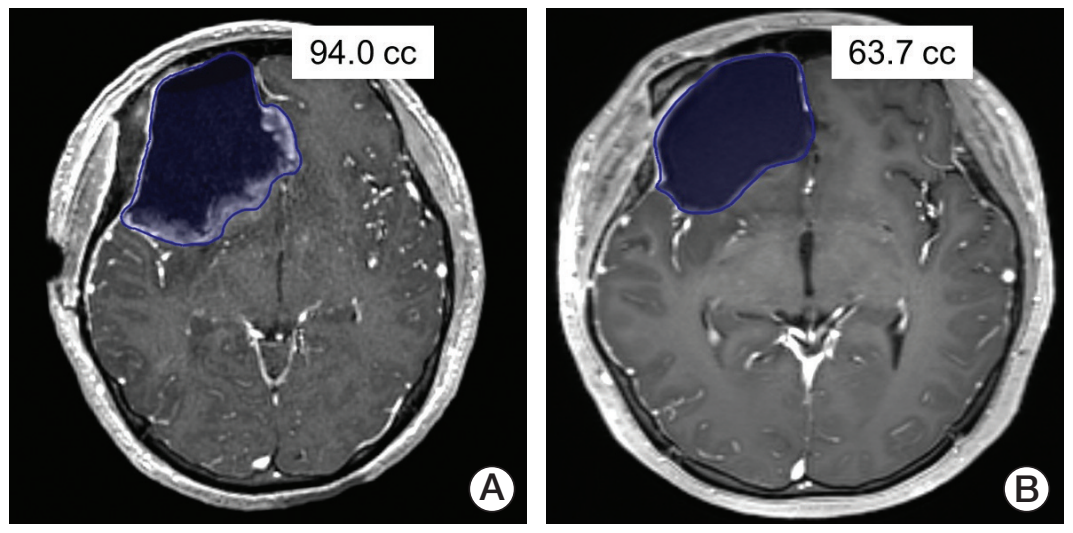

Fig. 1. A 23-year-old female with anaplastic oligodendroglioma in the right fronto-temporal lobe who underwent gross total resection of the tumor. Volumes of the contoured surgical cavities on postoperative (A) and pre-radiotherapy (B) magnetic resonance imaging are displayed.

\section{Volumetric changes of surgical cavities}

Of the 90 patients who underwent surgical resection, two MRIs each from 84 patients were able to be imported to the Eclipse RT planning system (ver. 13.7, Varian Medical Systems, Palo Alto, CA). No patient underwent any drainage procedure between the two MRIs. SCs on all MRIs were contoured by a single radiation oncologist (C.W.W.), and the volume (cc) was automatically measured via the system (Fig. 1).

We used two models to calculate the absolute daily change rate (ADCR). First, assuming an exponential change in the volume of SCs, the ADCR between the SCs on postoperative (post-op SC) and pre-RT (pre-RT SC) MRIs for each patient was calculated using the following equation:

$\left.\mathrm{ADCR}=\llbracket \mathrm{e}\left\{\frac{\mathrm{In}\left(\frac{\text { pre-RT SC volume (cc) }}{\text { post-op SC volume (cc) }}\right)}{\text { MRI interval (days) }}\right\}-1\right] \cdot 100(\% /$ day).

Second, we used a linear model to obtain the ACDR as the following: $\mathrm{ADCR}=\{$ (pre-RT SC volume [cc])-(post-op SC volume $[\mathrm{cc}])\} \times 100 /$ (post-op SC volume [cc])/(MRI interval [days]) (\%/day). The ADCR was obtained for each patient and the estimated change rates at postoperative 1, 2, 3, and 4 weeks were inversely calculated using the ADCR for each patient according to the exponential and linear models.

\section{Statistical analysis}

The Statistical Package for Social Sciences, ver. 22.0 (IBM Corp., Armonk, NY) was utilized for all statistical analyses. Fisher exact test was used to evaluate factors that might affect IPD. It was considered statistically significant when $\mathrm{p}<0.05$.

\section{Ethical statement}

The current study was performed under the approval from the Institutional Review Board of the Seoul National University Hospital (IRB No. H-1901-118-1005). The IRB has waived the need to obtain informed consent for this study. All research was performed in accordance with relevant guidelines/regulations.

\section{Results}

\section{Interim disease progression}

Among 121 patients, the median interval between the two MRIs was 23 days (interquartile range, 19 to 29 days). Only five patients $(4.1 \%)$ demonstrated IPD according to the aforementioned criteria. Three factors, which were biopsy (vs. surgical resection), presence of contrast-enhancing tumor on preoperative MRI, presence of remnant contrast-enhancing tumor on postoperative MRI, and the length of interval between MRIs were evaluated for their risk of IPD. Patients undergoing biopsy only and patients with remnant contrastenhancing lesion postoperatively had a significantly higher rate of IPD (Table 2). Of note, the mean interval of MRIs used for evaluation of IPD was significantly shorter in patients undergoing biopsy (20.7 \pm 8.4 days) compared to those receiving surgical resection $(28.2 \pm 18.4$ days) $(\mathrm{p}=0.032$ by independent $t$ test). However, the mean interval between MRIs did not 
Table 2. Risk factors for interim disease progression during the surgery-to-radiotherapy interval

\begin{tabular}{|c|c|c|c|c|}
\hline \multirow{2}{*}{ Variable } & \multirow{2}{*}{ No. } & \multicolumn{2}{|c|}{ IPD } & \multirow{2}{*}{ p-value ${ }^{a)}$} \\
\hline & & Yes & No & \\
\hline \multicolumn{5}{|l|}{ Surgery } \\
\hline Resection & 90 & $1(1.1)$ & $89(98.9)$ & 0.015 \\
\hline Biopsy & 31 & $4(12.9)$ & $27(87.1)$ & \\
\hline \multicolumn{5}{|l|}{ Preoperative T1CE lesion } \\
\hline Yes & 56 & $4(7.1)$ & $52(92.9)$ & 0.181 \\
\hline No & 65 & $1(1.5)$ & $64(98.5)$ & \\
\hline \multicolumn{5}{|l|}{ Postoperative remnant T1CE lesion } \\
\hline Yes & 102 & $2(2.0)$ & $100(98.0)$ & 0.027 \\
\hline No & 19 & $3(15.8)$ & $16(84.2)$ & \\
\hline Interval between MRIs (continuous, day) & & & & $0.876^{\mathrm{b})}$ \\
\hline
\end{tabular}

Values are presented as number (\%). IPD, interim disease progression; T1CE, contrast-enhancement of T1-weighted image.

${ }^{a}$ Fisher exact test (2-tailed), ${ }^{b}$ Logistic regression analysis.

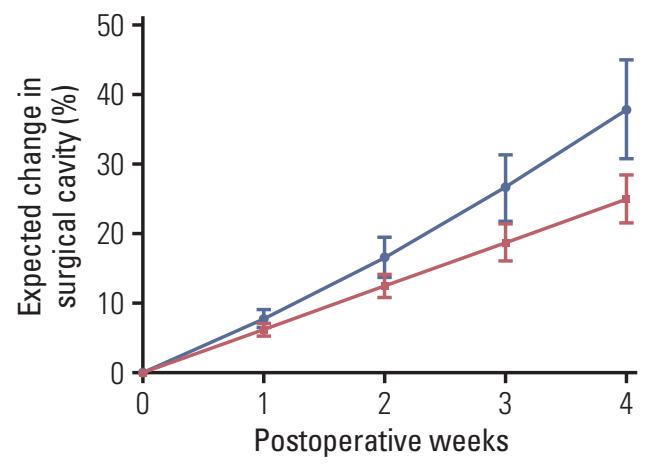

Fig. 2. Expected mean changes in the volume of postoperative surgical cavities in anaplastic glioma patients according to the exponential (blue line) and linear (red line) model ( $\mathrm{n}=84)$. Error bar, $95 \%$ confidence interval.

differ between patients with IPD (27.4 \pm 5.4 days) and without $\operatorname{IPD}(26.2 \pm 17.0$ days $)(\mathrm{p}=0.877)$.

\section{Volumetric changes of surgical cavities}

The median interval between two MRIs evaluable for analysis was 24 days (interquartile range, 19 to 30 days) for 84 patients undergoing surgical resection. Seventy-three $(86.9 \%)$ and 10 patients $(11.9 \%)$ demonstrated decreased and increased size of SCs, respectively. The SCs decreased and increased by a mean daily rate of $1.14 \%$ and $0.61 \%$ in those patients, respectively. One patient showed no change in the volume of SC. By the exponential model, the calculated mean ADCR of the SC was $1.06 \%$ (95\% confidence interval [CI], 0.89 to 1.23 ) for all patients. The expected mean absolute vol- umetric change of the SC was 7.83\% (95\% CI, 6.54 to 9.13), $16.64 \%$ (95\% CI, 13.77 to 19.52 ), $26.58 \%$ (95\% CI, 21.77 to 31.39), and $37.84 \%$ (95\% CI, 30.67 to 45.01 ) at postoperative $1,2,3$, and 4 weeks, respectively (Fig. 2). Assuming a linear change in the volume of SCs, the mean ADCR was 0.89\% (95\% CI, 0.77 to 1.02). The expected mean change of SCs at postoperative 1, 2, 3, and 4 weeks were $6.26 \%$ (95\% CI, 5.38 to 7.13 ), $12.51 \%$ (95\% CI, 10.77 to 14.26 ), $18.77 \%$ (95\% CI, 16.15 to 21.39 ), and $25.03 \%$ (95\% CI, 21.53 to 28.52 ), respectively (Fig. 2).

\section{Discussion}

AGs are a group of heterogeneous tumors which vary in histological/molecular characteristics, standard treatment, and prognosis [3-7]. However, RT is considered as standard therapy regardless of pathological diagnosis and type of surgery to date $[3,4]$. In order to perform RT precisely, accurate delineation of the RT-target is essential. Therefore, evaluation of the disease extent and the surrounding anatomical geometry at the time of RT planning is crucial. However, there has been no report evaluating the natural tumor growth as well as geometrical changes of the SC during the SRI in AGs.

The natural growth of glioblastoma evaluated by MRIs during the SRI has been studied by several investigators [9-12]. Pirzkall et al. [11] reported that increase in volume or new onset of contrast-enhancement occurred in $53 \%$ of glioblastoma patients during the SRI. Recently, VillanuevaMeyer et al. [12] have also reported that nearly half of glioblastoma patients show new or increased contrast-enhance- 
ment during the SRI. Furthermore, Wee et al. [9] adopted the size criterion from the RANO group [15], and evaluated the incidence of IPD during the SRI in the largest number of patients to date ( $n=166)$. They have reported that about $20 \%$ of patients demonstrate IPD and it leads to worsened survival in patients treated with chemoradiation [9].

In contrast to glioblastoma, IPD was rare in AG patients according to our results with an incidence below 5\%. Therefore, routine re-evaluation with brain MRI at the time of RT planning might not be beneficial for most of the AG patients. However, among these patients, patients who underwent biopsy or had residual contrast-enhancing lesions postoperatively were at an increased risk for IPD. These patients should be re-evaluated by MRI in the context of disease status before RT. In the current study, the risk of IPD was not evaluated based on histology (AA vs. AO vs. AOA) since except one patient, all patients $(n=30)$ who underwent biopsy alone had AA histology. Furthermore, the very one patient who was diagnosed as AOA and underwent biopsy did not show codeletion of $1 \mathrm{p} / 19 \mathrm{q}$ nor IDH mutation, in whom the diagnosis would have been AA according to the current World Health Organization classification [2]. Of note, no patient with AO histology demonstrated IPD during the SRI.

MRI is the recommended modality for evaluation of the brain tissues due to its superior resolution compared to CT scanning [16]. Fusion of diagnostic MRIs to RT planning CTs is a widely used method for accurate target delineation in various cancers, and it is most emphasized for brain tumors [5-7]. However, the optimal temporal sequence between brain MRIs and brain RT planning CTs for fusion is unknown, but it can be theoretically assumed that when taken on the same day, the two image sets would be identical regarding anatomical geometry. Therefore, we evaluated the volumetric change of the $\mathrm{SC}$, which is referred to as the gross tumor volume for AGs in most studies [5-7]. The estimated mean volumetric change of SCs was > 10\% after 2 weeks for $95 \%$ of the patients in both models in this study. This means if a radiation oncologist delineates the gross tumor volume based on the MRI taken 2 weeks before RT planning CT, there will be a mean discrepancy of approximately $15 \%$ com- pared to the gross tumor volume contoured on the MRI taken on the same day of RT planning CT. If the SC shrinks during the SRI, which would be the case in most patients, normal brain tissues can be over-irradiated unnecessarily. Conversely, if the SC expands due to any reason, the target brain tissues that need to be irradiated may receive suboptimal RT doses. Although this degree of change has not been validated whether it would affect RT planning significantly, the authors concluded that an additional brain MRI for accurate target delineation is necessary at the time of RT planning if the SRI exceeds 2 weeks, and it should be recommended in future clinical trials as well.

Obvious limitation of our study is the limited number of patients and the retrospective nature. The RANO criteria used in this study for evaluation of IPD is not a tool developed for assessment during the SRI [15]. Furthermore, the two models used for prediction of SC-change are not established models proven for its validity. The size of SC is expected to reach a plateau at some timepoint, which cannot be addressed by our study. Even in most of the patients who demonstrate increased size of SCs in the subsequent MRI, the $\mathrm{SC}$ are expected to decrease to some degree eventually. $\mathrm{Nev}$ ertheless, it was obvious in our study that volumetric changes in SCs during the SRI is notable during a short postoperative period, supporting the necessity for an additional planning MRI for RT.

We report the very first study evaluating IPD and volumetric change of SCs in AGs to the authors' knowledge. Unlike glioblastoma, IPD during SRI is rare in AG. However, additional MRIs at RT planning CT should be recommended for patients undergoing biopsy or demonstrating residual contrast-enhancement postoperatively in the context of disease evaluation. Similarly, in AG patients undergoing surgical resection, additional evaluation with MRI should be recommended since obvious change in the size of SC occur during the SRI.

\section{Conflicts of Interest}

Conflict of interest relevant to this article was not reported.

\section{References}

1. Ostrom QT, Gittleman H, Truitt G, Boscia A, Kruchko C, Barnholtz-Sloan JS. CBTRUS statistical report: primary brain and other central nervous system tumors diagnosed in the United States in 2011-2015. Neuro Oncol. 2018;20:iv1-86.

2. Louis DN, Perry A, Reifenberger G, von Deimling A, FigarellaBranger D, Cavenee WK, et al. The 2016 World Health Organ- ization classification of tumors of the central nervous system: a summary. Acta Neuropathol. 2016;131:803-20.

3. National Comprehensive Cancer Network. Central nervous system cancers (version 1.2019) [Internet]. Plymouth Meeting, PA: National Comprehensive Cancer Network; 2019 [cited 2019 Sep 9]. Available from: https://www.nccn.org/profes- 
sionals / physician_gls/pdf/cns.pdf.

4. Weller M, van den Bent M, Tonn JC, Stupp R, Preusser M, Cohen-Jonathan-Moyal E, et al. European Association for Neuro-Oncology (EANO) guideline on the diagnosis and treatment of adult astrocytic and oligodendroglial gliomas. Lancet Oncol. 2017;18:e315-29.

5. Cairncross G, Wang M, Shaw E, Jenkins R, Brachman D, Buckner J, et al. Phase III trial of chemoradiotherapy for anaplastic oligodendroglioma: long-term results of RTOG 9402. J Clin Oncol. 2013;31:337-43.

6. van den Bent MJ, Brandes AA, Taphoorn MJ, Kros JM, Kouwenhoven MC, Delattre JY, et al. Adjuvant procarbazine, lomustine, and vincristine chemotherapy in newly diagnosed anaplastic oligodendroglioma: long-term follow-up of EORTC brain tumor group study 26951. J Clin Oncol. 2013;31:344-50.

7. van den Bent MJ, Baumert B, Erridge SC, Vogelbaum MA, Nowak AK, Sanson M, et al. Interim results from the CATNON trial (EORTC study 26053-22054) of treatment with concurrent and adjuvant temozolomide for $1 \mathrm{p} / 19 \mathrm{q}$ non-co-deleted anaplastic glioma: a phase 3, randomised, open-label intergroup study. Lancet. 2017;390:1645-53.

8. Navarria P, Ascolese AM, Tomatis S, Reggiori G, Clerici E, Villa E, et al. Hypofractionated stereotactic radiation therapy in recurrent high-grade glioma: a new challenge. Cancer Res Treat. 2016;48:37-44.

9. Wee CW, Kim E, Kim TM, Park CK, Kim JW, Choi SH, et al. Impact of interim progression during the surgery-to-radiotherapy interval and its predictors in glioblastoma treated with temozolomide-based radiochemotherapy. J Neurooncol. 2017;134:169-75.

10. Pennington C, Kilbride L, Grant R, Wardlaw JM. A pilot study of brain tumour growth between radiotherapy planning and delivery. Clin Oncol (R Coll Radiol). 2006;18:104-8.

11. Pirzkall A, McGue C, Saraswathy S, Cha S, Liu R, Vandenberg $S$, et al. Tumor regrowth between surgery and initiation of adjuvant therapy in patients with newly diagnosed glioblastoma. Neuro Oncol. 2009;11:842-52.

12. Villanueva-Meyer JE, Han SJ, Cha S, Butowski NA. Early tumor growth between initial resection and radiotherapy of glioblastoma: incidence and impact on clinical outcomes. J Neurooncol. 2017;134:213-9.

13. Stensjoen AL, Solheim O, Kvistad KA, Haberg AK, Salvesen O, Berntsen EM. Growth dynamics of untreated glioblastomas in vivo. Neuro Oncol. 2015;17:1402-11.

14. Ellingson BM, Nguyen HN, Lai A, Nechifor RE, Zaw O, Pope $\mathrm{WB}$, et al. Contrast-enhancing tumor growth dynamics of preoperative, treatment-naive human glioblastoma. Cancer. 2016;122:1718-27.

15. Wen PY, Macdonald DR, Reardon DA, Cloughesy TF, Sorensen AG, Galanis E, et al. Updated response assessment criteria for high-grade gliomas: response assessment in neuro-oncology working group. J Clin Oncol. 2010;28:1963-72.

16. Leung D, Han X, Mikkelsen T, Nabors LB. Role of MRI in primary brain tumor evaluation. J Natl Compr Canc Netw. 2014; 12:1561-8. 